\title{
Werner Grünzweig, Wie entsteht dabei Musik? Gespräche mit sechs Komponisten und einer Komponistin über ihre Studienzeit, Neumünster: von Bockel 2019
}

Schlagworte/Keywords: Bernhard Lang; composition and music theory; Enno Poppe; Georg Friedrich Haas; Gösta Neuwirth; Hanspeter Kyburz; Interviews; Isabel Mundry; Komposition und Musiktheorie; Orm Finnendahl; Peter Ablinger

\section{KONTEXT}

Der an fast jeder größeren Musikhochschule im deutschsprachigen Raum angebotene Kompositionsunterricht ist ein Bereich der künstlerischakademischen Lehre, welcher für den externen Blick oftmals verschlossen bleibt. Innerhalb der Hochschule ist das bereits durch das größenbedingte Nischendasein der Kompositionsstudiengänge mitbedingt, und auch die vorherrschenden Lehrformate - studiengangsinterne Kleingruppen und, besonders im Hauptfach, Einzelunterricht - tragen nicht zur Durchlässigkeit des Lehrbetriebs bei. Daneben ist aber auch das Fehlen eines breiteren, von außen zugänglichen Diskurses über Ansätze, Methoden und Praktiken des Kompositionsunterrichts zu verzeichnen. ${ }^{1}$ Das verwundert nicht nur angesichts der langen Tradition institutionalisierter Kompositionslehre, sondern auch wegen der signifikanten Rolle, welche dem Kompositionsstudium in den individuellen Karrieren junger Komponist*innen nach wie vor zukommt. Denn trotz der in letzter Zeit beobachtbaren Tendenz, im Zuge zunehmender Transund Interdisziplinarität auch traditionell außerakademisch verortete Musikszenen, -stile und -praktiken in die Sphäre der neuen Musik mit einzubeziehen, so ist ein abgeschlossenes Kompositionsstudium doch nach wie vor eine hilfreiche, teils sogar notwendige Voraussetzung, um Zugang zur institutionellen Konzert-, Festival- und Förderkultur zu erhalten.

Ausnahmen bilden hier musikpädagogische Veröffentlichungen auf dem Gebiet der Vermittlungspraxis neuer oder experimenteller Musik zumeist in schulischen Kontexten, vgl. Schlothfeldt 2015; Wieneke 2016; Schlothfeldt/Vandré 2018.
Vor diesem Hintergrund erscheint der Band Wie entsteht dabei Musik? Gespräche mit sechs Komponisten und einer Komponistin über ihre Studienzeit mit dem Versuch, »eine Innenansicht davon [zu vermitteln], was es bedeutet, Komposition zu studieren « (so der Klappentext) als eine lohnenswerte Unternehmung, um dem interessierten Blick von außen erhellende Einsichten in die Sphäre des akademischen Kompositionsunterrichts zu gewähren und so potenzielle Anknüpfungspunkte an ganz unterschiedliche Diskurse (kompositionstheoretische, musikpädagogische, historiographische, hochschulpolitische) aufzuzeigen. Eine zusätzliche, explizit für die musiktheoretische Rezeptionsperspektive relevante Dimension ergibt sich aus dem personellen Gravitationszentrum der sieben im Band versammelten Interviews, welche Werner Grünzweig, der langjährige Leiter der Musikarchive der Berliner Akademie der Künste, mit sechs Komponisten und einer Komponistin geführt hat. Denn alle sieben Gespräche umkreisen - mal entfernter, zumeist aber in unmittelbarer Nähe - das Wirken des 1937 geborenen Komponisten und Musiktheoretikers Gösta Neuwirth. Genauer gesagt, geht es um Neuwirths Wirken als Hochschullehrer und insbesondere seine Rolle als "alternativer Kompositionslehrer « (Klappentext), welche alle sieben Interviewten in je eigener Weise als prägend erlebt haben. Als besonders bemerkenswert kann das insofern gelten, als Neuwirth nie als institutioneller Kompositionslehrer tätig war, sondern offiziell - zuerst in Graz und von 1983 bis 2002 als Professor an der Universität der Künste (vormals Hochschule der Künste) Berlin - ausschließlich Musiktheorie unterrichtete. Gleichwohl übte er auf eine Vielzahl von Kompositionsstudierenden - die im Band 
interviewten Peter Ablinger, Orm Finnendahl, Georg Friedrich Haas, Hanspeter Kyburz, Bernhard Lang, Isabel Mundry und Enno Poppe bilden hier nur eine Auswahl besonders prominenter Schüler*innen - einen Einfluss aus, dessen Wirkung mit jener des Kompositionshauptfachunterrichts vergleichbar war oder diese sogar noch übertraf. Aus musiktheoretischer Sicht interessant erscheint also die Frage, wie genau sich das von Neuwirth verkörperte enge Wechselverhältnis von Musiktheorie und Kompositionslehre darstellte und ob eine produktive Übertragung in die heutige Zeit möglich und wenn ja - wünschenswert ist.

\section{AUFBAU}

Der Textteil des Bandes umfasst sieben Kapitel, die jeweils ein Komponist*innen-Gespräch enthalten, und wird eingeleitet von einem kurzen Vorwort Grünzweigs. In diesem erfährt man unter anderem, dass die Gespräche bereits in den Jahren 2007 und 2008 geführt wurden, dass sie Grünzweig allerdings erst 2017 für eine Veröffentlichung in Erwägung zog. Ob es sich in der nun veröffentlichten Form um die gesamten Gespräche handelt, inwieweit sie gekürzt oder nach welchen Kriterien Teile ausgewählt wurden, bleibt dagegen leider unklar. Offenkundig hingegen ist, dass die Überarbeitungen der (bis auf ganz wenige Flüchtigkeitsfehler) sehr sorgfältig edierten Gesprächstranskriptionen sehr dezent ausgefallen sind, denn der jeweilige individuelle Tonfall der Interviewpartner*innen vermittelt sich ebenso unmittelbar wie die naturgemäß teils assoziative, sprunghafte oder abschweifende Dramaturgie des Interviewformats.

Bei der Anordnung der sieben Gesprächskapitel scheint sich Grünzweig primär chronologisch am Geburtsjahr seiner Interviewpartner*innen orientiert zu haben. So gehören die sieben interviewten Komponist*innen - vom 1953 geborenen Georg Friedrich Haas bis zum jüngsten vertretenen Neuwirth-Schüler, dem 1969 geborenen Enno Poppe, reichend - mindestens drei unterschiedlichen Studierendengenerationen an. Die im Vorwort formulierte These Grünzweigs, dass Neuwirth »zu den einflußreichsten Kompositionslehrern seiner Zeit zu zählen ist « (7) wird aber nicht nur durch diese temporale Breite und die Promi- nenz der Namen gestützt, ebenso unterstreicht die bemerkenswerte stilistische Diversität der sieben Komponist*innen Neuwirths Einfluss. So sind etwa mit Peter Ablingers Konzeptualismusansatz, Haas' Mikrotonalitätsaneignung oder Bernhard Langs Loop-Musik ausgesprochen unterschiedliche Denkschulen und Traditionslinien in Neuwirths Schülerschaft vertreten. Schon durch Selektion und formale Zusammenstellung seiner Interviewpartner weist Grünzweig also auf die im Verlauf der Gespräche auch inhaltlich thematisierte Qualität von Neuwirths pädagogischem Wirken hin, in Richtung ganz unterschiedlicher ästhetischer Dispositionen und Präferenzen seine Wirkung entfaltet zu haben.

\section{PERSPEKTIVITÄT}

Die grundlegende, im Vorwort explizit reflektierte Entscheidung Grünzweigs, die sieben Gespräche unkommentiert nebeneinandergestellt zu präsentieren, erzeugt im Resultat eine Multiperspektivität, welche sowohl für den informativen Gehalt der einzelnen Gespräche wie auch für die Leseerfahrung und Wirkung des Bandes als Ganzes konstitutiv ist. Auf inhaltlicher Ebene bildet die Person Neuwirths das gemeinsame Objekt, auf das die sieben Interviewten - durch Grünzweigs Fragestellungen mal mehr, mal weniger gezielt gelenkt ihren subjektiven Fokus richten. Neben den naturgemäß individuell unterschiedlichen Wahrnehmungen, Meinungen und Wertungen, die in das jeweils gezeichnete Neuwirth-Porträt einfließen, ist es dabei auch die zeitliche Komponente, welche das entstehende Gesamtbild weiter auffächert. Denn einerseits wird von seiner Anfangszeit in Graz, in die Georg Friedrich Haas' und Peter Ablingers Bekanntschaft mit Neuwirth fällt, bis zum Ende des Studiums von Enno Poppe, als Neuwirth kurz vor seiner Emeritierung stand, fast dessen gesamte Hochschulkarriere in den zeitlichen Horizont mit einbezogen, andererseits schreibt sich unweigerlich auch die bekanntermaßen kreative Dynamik des Gedächtnisses in die zum Gesprächszeitpunkt teils über 30 Jahre zurückliegenden Erinnerungen mit ein. Zusammengenommen mit dem zehnjährigen Intervall zwischen Interviews und Veröffentlichung ergibt sich mithin eine multiple zeitliche Brechung, 
welche die Autorität eines einheitlichen, umfassenden Bildes oder Narrativs von vornherein in Frage stellt. Ganz ähnlich wie in Akira Kurosawas berühmtem multiperspektivisch erzählten Rashomon (1950), einem Lieblingsfilm Neuwirths, auf den er auch in seinem Unterricht immer wieder zu sprechen kam, ist man als Leser*in dadurch automatisch dazu herausgefordert, auf Zusammenhänge und Bezüge zwischen den unterschiedlichen Darstellungen zu achten und aus den angebotenen Sichtweisen ein eigenes Bild zu extrahieren. In manchen Fällen gelingt das problemlos - über Neuwirths eben erwähnte Referenz auf japanische Filmkunst z. B. wird einhellig berichtet -, in anderen Fällen - besonders was die Bewertung von Neuwirths Lehrerrolle und sein Verständnis von Musiktheorie angeht - gilt es hingegen immer wieder auch, voneinander abweichende Positionen und komplementäre Informationen miteinander abzustimmen. Eine solche aktiv-konstruierende Lesehaltung kann freilich zur Selbstreferentialität tendieren (und ist daher normalerweise auch eher in literarischen Kontexten zu finden); im vorliegenden Fall steht sie allerdings ganz im Dienst der Gesamtwirkung. Diese kann sich erstaunlich effektiv entfalten, einerseits weil die Gespräche eine ausgewogene Balance aus Kon- und Divergenzen, aus offensichtlichen Sinnangeboten und versteckten Interpretationsaufforderungen bieten und andererseits weil die Herausforderung des sinnstiftenden Leseakts die - im Vergleich zu einem konzise ausformulierten schriftsprachlichen Text - naturgemäß relativ geringe bzw. sehr schwankende Informationsdichte aufmerksamkeitsökonomisch kompensiert. Durch die im Laufe der Kapitel immer deutlicher werdende multiperspektivische Grunddisposition erhält der Band, der zunächst noch als leichte, in flüssigem Konversationston gehaltene Lektüre erscheint, mithin eine anfangs nicht erahnte infratextuelle Tiefendimension, welche ganz wesentlich zur Lust am Text beiträgt.

\section{VIELSCHICHTIGKEIT}

Die skizzierte Multiperspektivität bietet nicht nur den kohärenzstiftenden formalen Rahmen der sieben Gespräche, sie begünstigt auch eine inhaltlich vielschichtige Leseweise des Bandes.
Denn dadurch, dass sich als Fokus der Gespräche ganz klar die Konturierung eines mosaikartigen Neuwirth-Porträts herauskristallisiert, kann der sinnstiftende Leseakt je nach Individualinteresse einzelne Aspekte - sowohl innerhalb als auch außerhalb dieses Rahmens - in den Vordergrund treten lassen, miteinander verbinden oder auch vollkommen ausblenden. So wird z. B. jemand, der sich für das österreichische Musikhochschulwesen der 1970er Jahre interessiert, in den Gesprächen mit Peter Ablinger, Bernhard Lang und Georg Friedrich Haas, in denen diese schildern, wie sie Neuwirth als eine der wenigen Personen an der ansonsten eher regressiven Grazer Musikhochschule kennen und schätzen lernten, sicher die eine oder andere neue Information finden. Nichtexperten hingegen werden diesbezügliche Details, Personen und auch manches Anekdotische einfach überlesen. Ebenso kann man - das jeweilige Interesse vorausgesetzt ohne Frage auch Aufschlussreiches über individuelle Lebensläufe der interviewten Komponist*innen und über konkrete damit in Verbindung stehende historische Gegebenheiten erfahren, so etwa wenn Isabel Mundry stimmungsvoll von ihrer musikalischen Sozialisation im West-Berlin der 1980er Jahre erzählt und z. B. von der Schwierigkeit berichtet, überhaupt einen qualifizierten Privatlehrer für Komposition zu finden. Und auch kompositionsmethodisch Interessierte werden immer wieder auf eingestreute Details stoßen, etwa dass offenbar noch vor zwei Lehrendengenerationen (namentlich bei Frank Michael Beyer an der Hochschule der Künste) ein an kontrapunktischen Exempla und Praecepta orientierter, aus heutiger Sicht antiquiert wirkender Unterricht im Hauptfach Komposition praktiziert wurde. Doch sieht man von solchen oder anderen eher von Partikularinteressen geleiteten Perspektiven $a b$, so werden die meisten Leser*innen die genannten Einzelaspekte doch wohl primär eher als Hintergrund für das wahrnehmen, was sich ohne Frage in den Vordergrund des Sichtfeldes drängt: die Person und das Wirken Gösta Neuwirths.

Versucht man, aus einer übergeordneten, um Ausgewogenheit bemühten Perspektive heraus die unterschiedlichen Sichtweisen auf Neuwirth zusammenzubringen, so ergibt sich ein komplexes, vielschichtiges Bild, auf dessen 
Oberfläche gleichwohl einige Merkmale deutlich hervortreten: so etwa die vorurteilslose Offenheit und das aufrichtige Interesse, das Neuwirth gegenüber allen aufbrachte, die sich ernsthaft mit der künstlerischen Moderne im Allgemeinen und der neuen Musik im Besonderen beschäftigen wollten, und - damit verknüpft - die großzügige Bereitschaft, für diese Interessierten ein Ausmaß an Zeit und Energie aufzubringen, welches den Rahmen der Lehrverpflichtungen regelmäßig sprengte und daher oft in außerakademische, halb-private, teils auch freundschaftliche Formate kanalisiert wurde. Zu nennen wäre aber auch Neuwirths auf den ersten Blick alles andere als charismatische, sondern vor allem ein Höchstmaß an Intellektualität und zuweilen auch Zaudern und Skepsis ausstrahlende Erscheinungs- und Ausdrucksweise, welche aber offenbar immer wieder eine ungeahnt enthusiastische Intensität zu vermitteln in der Lage war. Das hing wohl vor allem auch mit Neuwirths spezifischer Art zusammen, von jenen persönlichen, teils recht spezifischen Interessen auszugehen, von denen auch die übersichtliche Zahl seiner fachbezogenen Publikationen zeugen (vor allem sind hier Franz Schreker, die Wiener Schule und die franko-flämische Vokalpolyphonie zu nennen), diese aber immer auch schon zu entpersonalisieren und, teils extrem assoziativ und spekulativ, in größere, die Grenzen von Musiktheorie und Komposition sprengende Kontexte einzubetten. Die virtuose Trans- bzw. Interdisziplinarität dieses scheinbar paradoxen, objektiviertindividuellen, domestiziert-wilden Denkens wurde von vielen Kompositionsstudierenden insbesondere wenn ihr Hauptfachunterricht eng umgrenzt und vor allem auf handwerkliche Fertigkeiten ausgerichtet war - als eine befreiende Horizonterweiterung erfahren, weshalb sich viele von ihnen immer wieder auch mit eigenen kompositorischen Arbeiten an Neuwirth wandten. Die Faszinationskraft Neuwirths konnte so eine Dynamik entfalten, welche in einer bestimmten Phase der 1980er Jahre zu einer sich aus Neuwirths Theorieseminaren herausbildenden Gruppe führte, welche für Außenstehende zuweilen als esoterischsektenhaft wahrgenommen wurde. Diese Wirkung hatte wohl nicht zuletzt auch mit einer nach außen gerichteten Abgrenzung zu tun, welche mit der Übernahme von Neuwirths grundlegend querständig-rebellischer Haltung gegenüber institutionellen Vorgaben und Zwängen zusammenhing.

Wenn die hier sehr grob zusammengefassten Grundzüge sich - in unterschiedlicher Akzentuierung - durch alle sieben Gespräche ziehen, so weichen die Darstellungen anderer Aspekte in den Augen von Neuwirths früheren Schüler*innen doch auch signifikant voneinander ab. Diese Varianzen lassen sich einerseits durch unterschiedliche zeitliche Perspektiven auf, im Grunde genommen, konstante Merkmale von Neuwirths Wirken erklären. Während z. B. Haas und Ablinger davon erzählen, dass sie Neuwirth in seiner Grazer Zeit als Verfechter einer sechten ‘ Avantgarde kennenlernten, der aus einer selbstbewusst-kritischen Perspektive heraus aufgezeigt habe, dass "man die Sachen überhaupt auseinandernehmen kann, die erstmal so kompakt daherkommen « (48), so berichtet Orm Finnendahl aus seiner ca. 15 Jahre später gewonnenen Perspektive heraus dagegen von seinem Gefühl, dass "Gösta viel von der Entwicklung der zeitgenössischen Künste nicht mitbekommen hat, aber in seinem Selbstverständnis ständig auf der Höhe seiner Zeit war" (162); und Isabel Mundry hinterfragt nicht zuletzt auch von ihrer Position als Kompositionsprofessorin aus, ob Neuwirths unbedingter Anspruch auf kompositorische Autonomie und künstlerische Unkorrumpierbarkeit den Studierenden nicht auch ein problematisches, heutzutage nicht mehr legitimierbares Beispiel potenzieller Kommunikationsverweigerung gegeben habe (134-141). Scheinbar relativ konstante Auffassungen, Interessen und Eigenschaften Neuwirths werden hier also aus unterschiedlichen Perspektiven ganz verschieden gesehen. Andere Abweichungen sind dagegen viel stärker durch die Beziehungsarten und -intensitäten zu Neuwirth sowie die unterschiedlichen Persönlichkeiten der ehemaligen Schüler*innen bedingt. Das bezieht sich vor allem auf den Gestus der abwägenden Distanznahme. Denn wenn die positiv prägenden Einflüsse Neuwirths in der Darstellung von allen sieben Interviewten auf ähnliche Qualitäten des Lehrers zurückgeführt werden - etwa seine analytische Geistesschärfe, assoziative Originalität und antiautoritäre Art -, so unterscheiden sich die kritischen Auseinandersetzungen mit Neuwirth doch recht stark. Am 
besten lässt sich das an den Darstellungen Mundrys und Kyburz' ablesen, beide Angehörige des oben erwähnten inneren, GöstaZirkels` in Neuwirths früher HdK-Zeit.

Mundrys bereits oben skizzierte teils kritische Beurteilung ist nicht nur sehr differenziert gehalten, sie basiert auch auf der explizit selbstreflektierten Beschreibung einer allmählichen Entzauberung von Neuwirths Faszinationskraft auf sie. Die Kritik Hanspeter Kyburz' hingegen fällt deutlich prinzipieller aus. So schildert dieser zwar einerseits einen ähnlichen Relativierungsprozess, wenn er vor allem das zeitgleich begonnene Studium bei Carl Dahlhaus an der Technischen Universität dafür verantwortlich macht, dass viele spekulative Thesen Neuwirths, die zunächst »unglaublich interessant wirkten, [...] sich später als leer erwiesen « (94 f.), gleichzeitig unterstellt er Neuwirth aber auch eine gezielte Verunklarungsstrategie: „Er [Neuwirth] hat Dinge auch bewußt spekulativ dargestellt. Und diese produktive Verwirrung bekam irgendwann etwas Habituelles, Stilisiertes, auch Selbststilisiertes [...].« (82) Eine solche sich am Ideal objektiver Darstellungsweise und überzeitlicher Geltung orientierende Problematisierung ist ohne Frage legitim und - setzt man einen wissenschaftlichen Maßstab an Neuwirths Thesen an - auch geboten (ein gutes Exemplifikationsobjekt dafür stellt z. B. Neuwirths Artikel über gematrisches Denken bei Josquin des Prez dar) ${ }^{2}$, gleichzeitig verfehlt sie aber auch das Moment situativsuggestiver Inspiration, welches laut Kyburz so charakteristisch für Neuwirths Unterrichtsweise war und - so ist den anderen Gesprächen zu entnehmen - auf die Mehrzahl der Studierenden auch vollkommen authentisch gewirkt hat. $^{3}$

2 Vgl. Neuwirth 1982.

3 Kyburz' unausgewogene Kritik ist vielleicht aus seiner intensiven Involvierung in die Einflusssphäre Neuwirths zu erklären. So wurde er wie sich aus den Gesprächen mit Bernhard Lang und Orm Finnendahl ergibt - unter allen Mitgliedern des `Gösta-Zirkels` aus der Außenperspektive als Neuwirths glühendster Anhänger angesehen - eine Rolle, die er in seinem eigenen Interview bezeichnenderweise gänzlich ausklammert.

\section{ÜBERTRAGBARKEIT}

Kyburz' Kritik berührt gleichwohl einen neuralgischen Punkt, der für eine musiktheoretische Rezeptionsperspektive, die nach der Relevanz und potenziellen Übertragbarkeit von Neuwirths Hochschulwirken fragt, von entscheidender Bedeutung ist. Er betrifft gleichermaßen das Selbstverständnis wie die Außenwahrnehmung Neuwirths im Spannungsverhältnis zwischen künstlerisch-assoziativ-generativem und wissenschaftlich-universalistisch-exaktem Denken. Isabel Mundry fasst das sehr schön zusammen, wenn sie Neuwirth als jemanden beschreibt,
der auf sehr eigenwillige Weise die Dinge so in Beziehung zueinander setzt, daß sie für ihn sinnhaft werden und aus dieser Perspek- tive auch für andere interessant sind. Das sind Bereiche gewesen, die je einen institu- tionell eigenen Ort hatten, aber selten zu- sammenkamen. Gösta ist im Grund inter- oder transdisziplinär in sich selbst [...], er hat sich erlaubt, den Umgang mit Musikwissen- schaft und Theorie nicht als institutionelle Vorgabe zu betrachten [...], sondern als eine Möglichkeit, die man selbst bespielt. (133)

Wesentliche Kernaspekte der Lehrergestalt Neuwirth scheinen hier noch einmal zusammengefasst auf: der persönliche Zugriff und Enthusiasmus für die Sache, die Lizenz zum selbstbestimmten Denken, die Modernität grenzüberschreitender Zusammenhangsbildungen, die Faszinationskraft, welche nicht nur auf Wissen, sondern auch auf Suggestion und konstruktiver Spekulation beruht. Einige dieser Merkmale sind nun ohne Frage Qualitäten, die auch für die heutige musiktheoretische Praxis in Forschung wie Lehre Maßstäbe abgeben könnten. Wenn z. B. Enno Poppe eindrücklich beschreibt, dass es Neuwirth selbst bei der Korrektur simpler Kontrapunktaufgaben stets darum ging, dass wauch in den einfachsten Dingen immer Ideen verborgen sind" (175), dann drückt sich darin ein von künstlerischkompositorischer Praxis durchdrungener Ansatz aus, der für einen inspirierten musiktheoretischen Unterricht als nach wie vor vorbildhaft gelten kann. Andere Aspekte hingegen erscheinen problematischer. So sollte sich die gegenwärtige Musiktheorie, gerade weil sie immer noch zwischen künstlerischer Scylla und wissenschaftlicher Charybdis manövriert, vielleicht 
weniger mithilfe eines vagen, assoziativspekulativen Richtungssinns als vielmehr anhand eines klaren Methodenkompasses orientieren. Folglich gälte es, wissenschaftliche und künstlerische (inklusive spekulativer) Ansätze und Paradigmen in ihrem Methodenrepertoire zunächst einmal klar auseinanderzuhalten und - wenn gewünscht - explizit gekennzeichnet miteinander in Beziehung zu setzen. Georg Friedrich Haas' mit offensichtlichem Bedauern geäußerte Bemerkung, dass es in der heutigen Hochschullandschaft keinen Platz mehr für eine Lehrergestalt wie Gösta Neuwirth gäbe (vgl. 23), wäre in dieser Hinsicht daher auch zu relativieren. Denn die Besetzung einer Professur für die Geschichte der Musiktheorie (diese hatte Neuwirth in Berlin inne) mit jemandem, der fast ausschließlich systematisch und spekulativ argumentiert, wäre in Zeiten historisch informierter Musiktheorie mit gutem Recht undenkbar.

Abgesehen von den oben skizzierten allgemeinen berufsethischen und persönlichkeitsbezogenen Qualitäten erscheint eine Übertragung von Neuwirths Unterrichtspraxis auf heutige Verhältnisse also schwierig, nicht zuletzt auch, weil sich die hochschulpolitischen Rahmenbedingungen so grundlegend geändert haben, dass die Lizenzen der Unterrichtsgestaltung, die sich Neuwirth gestattete, im heutigen durchorganisierten Bachelor- und Mastersystem vollkommen unmöglich erscheinen. Gleiches gilt - auch wenn hier die Freiheiten im künstlerischen Hauptfachunterricht immer noch größer sein mögen - auf dem Feld der Kompositionslehre. Dass der Band hier eher allgemeine als methodisch konkrete Anregungen liefern kann, liegt einerseits daran, dass Neuwirth in seiner Rolle als salternativer Kompositionslehrer seine Wirkungskraft oftmals gerade durch die Komplementarität zum regulären Kompositionsunterricht entfalten konnte, während ein ausschließlicher Hauptfachunterricht ohne Frage ganz eigene Anforderungen stellt; andererseits erfolgen die in den einzelnen Gesprächen enthaltenen Diskussionen kompositionstechnischer Fragestellungen formatbedingt ohne Anschauungsmaterialien wie Partituren oder ähnliches und sind deswegen schlicht zu abstrakt gehalten, als dass genauere methodische oder systematische Schlüsse aus ihnen gezogen werden könnten. Der im Titel des Bandes dem Gespräch mit Enno Poppe entnommenen Frage, wie im und durch den Kompositionsunterricht Musik entstehe, kann der Band daher nur andeutungsweise beikommen. Die versprochene Einsicht ins Innenleben des Kompositionsstudiums aus den persönlichen, größtenteils informativen, teils auch anekdotischen Erinnerungen der interviewten Komponist*innen ist gleichwohl höchst lesenswert und teilweise auch recht unterhaltsam. Der Hauptverdienst des Bandes liegt aber ohne Frage in der multiperspektivisch-historiographischen Rekonstruktion einer fiktiven 'Klasse Neuwirth. Mit dieser vielstimmig aufgefächerten Hommage an eine beeindruckende Lehrergestalt gelingt Grünzweig eine Fortschreibung seines bereits 1997 zum 60. Geburtstag Neuwirths publizierten Sammelbandes zu dessen Schaffen. Anders als dort kommt Neuwirth selbst allerdings nicht zu Wort, vielmehr ist er allein durch eingestreute Manuskriptseiten einzelner Kompositionen vertreten. Diese editorische Geste passt sich stimmig in das ungewöhnliche Format des Bandes ein und trägt dazu bei, dass eine von Isabel Mundry beiläufig geäußerte, aber vollkommen zutreffende Forderung eingelöst wird, nämlich dass eine angemessene Rezeption des Wirkens von Gösta Neuwirth »genauso eigenwillige Formen entwickeln muß, wie sein Schaffen eigenwillig ist« (135).

Tom Rojo Poller 


\section{Literatur}

Grünzweig, Werner (Hg.) (1997), Gösta Neuwirth. Verzeichnis der musikalischen Schriften und Editionen, Inventar der Musikalien im Gösta-Neuwirth-Archiv, Hofheim: Wolke.

Neuwirth, Gösta (1982), „Erzählung von Zahlen«, in: Josquin des Prés (= Musik-Konzepte, Bd. 26/27), hg. von Heinz-Klaus Metzger und Rainer Riehn, München: edition text + kritik, 4-38.
Schlothfeldt, Matthias (2015), Komponieren im Unterricht, 2. Auflage, Hildesheim: Olms.

Schlothfeldt, Matthias / Philipp Vandré (Hg.) (2018), Weikersheimer Gespräche zur Kompositionspädagogik, Regensburg: ConBrio.

Wieneke, Julia (Hg.) (2016), Zeitgenössische Musik vermitteln in Kompositionsprojekten an Schulen, Hildesheim: Olms.

Poller, Tom Rojo (2020): Werner Grünzweig, Wie entsteht dabei Musik? Gespräche mit sechs Komponisten und einer Komponistin über ihre Studienzeit, Neumünster: von Bockel 2019. ZGMTH 17/1, 207-213. https://doi.org/10.31751/1038

(C) 2020 Tom Rojo Poller (trp@trpoller.de)

Universität der Künste Berlin [Berlin University of the Arts]

Dieser Text erscheint im Open Access und ist lizenziert unter einer Creative Commons Namensnennung 4.0 International Lizenz.

This is an open access article licensed under a

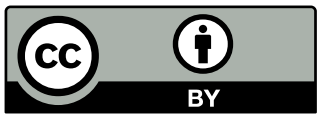

Creative Commons Attribution 4.0 International License.

eingereicht / submitted: 15/01/2020

angenommen / accepted: 15/01/2020

veröffentlicht / first published: 15/06/2020

zuletzt geändert / last updated: 15/06/2020 